\title{
AN INVESTIGATION OF THE MAXIMUM EXPERIMENTAL SAFE GAP ANOMALY*
}

\author{
ROGER A. STREHLOW** \\ Aeronautical and Astronautical Engineering Department, University of Illinois at \\ Urbana-Champaign, Urbana, IL (U.S.A.)
}

\section{J. ARTHUR NICHOLLS}

Department of Aerospace Engineering, University of Michigan, Ann Arbor, MI (U.S.A.)

ERNEST C. MAGISON

Honey well, Inc., Fort Washington, PA (U.S.A.)

and PETER J. SCHRAM

Underwriters' Laboratories, Inc., Northbrook, IL (U.S.A.)

(Received June 5, 1978)

\section{Summary}

The "safe gap", is defined as the maximum gap between two close-fitting, flat, machined metal surfaces that will just prevent an explosion within a vessel from producing a propagating combustion wave in the gases that surround the vessel. At present three distinctly different apparatuses are used throughout the world to determine experimentally the maximum safe gap for different combustible gases and vapors. Unfortunately, the maximum safe gap thus determined sometimes differs widely from vessel to vessel. This report investigates the nature of the processes by which the explosion is transmitted to the surrounding media, paying particular attention to the differences from apparatus to apparatus. It is found that the time constants associated with the combustion process and with vessel exhausting are markedly different for the different vessels and that the pressure levels developed inside a vessel during the test also vary markedly from vessel to vessel. Based on these observations, it is recommended that a more thorough theoretical and experimental investigation of the safe gap testing procedures and vessel design be made as the basis for a proper design for adequate safe gap testing of all combustible vapors and gases.

\section{Introduction}

In many industrial and some commercial locations flammable gases and vapors exist in concentrations that can propagate flames. In many cases electrical equipment must also be used and operated in these areas, thus presenting a hazard because of the possibility that an electrical arc or spark or parts operating at high temperatures will cause ignition and subsequent fire or explosion of the flammable mixture in the work space.

\footnotetext{
*Letter report of a panel of the Committee on Industrial Hazards, National Materials Advisory Board, Commission on Sociotechnical Systems, National Academy of Sciences. The Committee is chaired by Dr. Homer Carhart.

**Chairman of panel.
} 


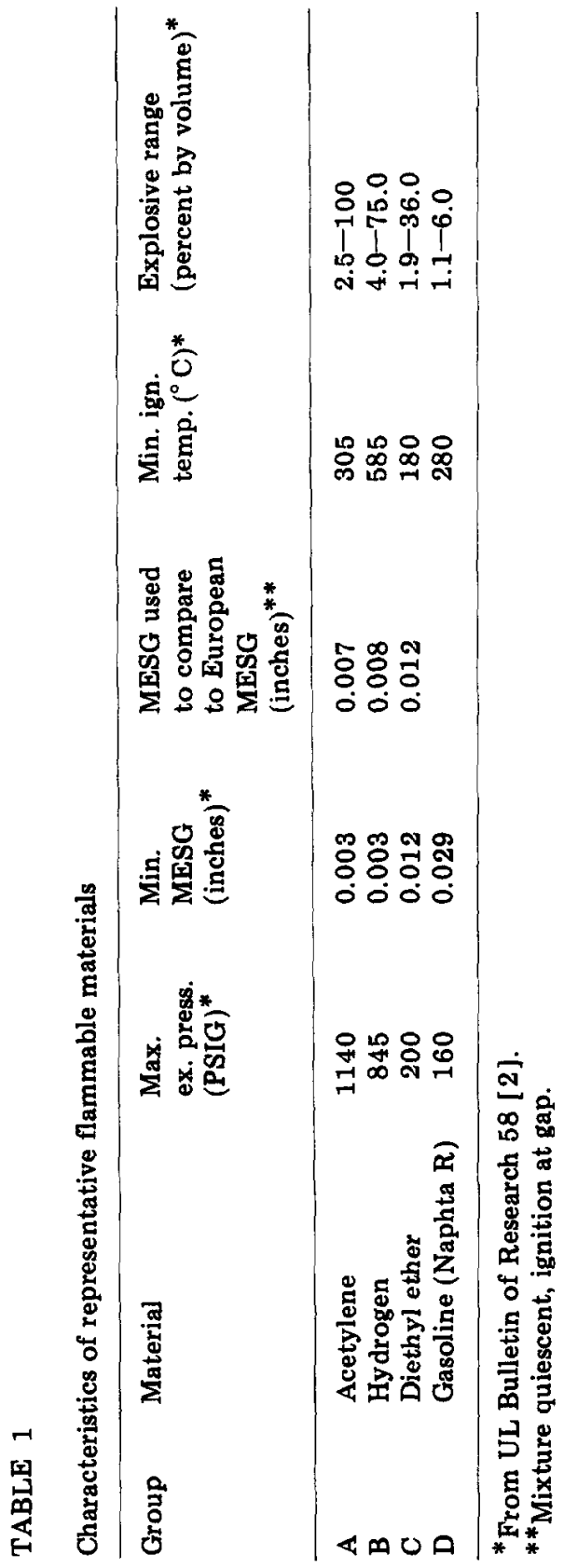


A major form of protection against this hazard in the United States has been to contain all such electrical equipment in enclosures constructed so that even if an explosion occurs within the enclosure due to ignition of the flammable contents, the subsequent internal explosion will not be propagated to the surroundings, either through any openings in the enclosure or by failure of the enclosure itself. Openings in the enclosure, even though small, are necessary. They are needed to permit assembly, installation, and maintenance of the equipment. For some equipment, such as motors and manually operated switches, operation of the equipment requires penetration of the enclosure by a moving part. Because of these openings, the flammable gases and vapors that may surround the enclosure cannot be prevented from entering the enclosure. Such enclosures are usually referred to as "explosion-proof enclosures". There are other methods of protection for electrical equipment in flammable atmospheres, but these methods are not related to the anomaly covered by this report.

The National Electrical Code [1] classifies flammable gases and vapors into four groups, A, B, C, and D, based on the explosion pressure of the material and the maximum gap between two close-fitting, flat, machined metal surfaces that will just prevent an explosion within a vessel from producing a propagating combustion wave in the gases surrounding the vessel. The gap is the maximum experimental safe gap (MESG).

Since 1968 in the United States the classification of flammable gases and vapors into the four groups has been based on tests in the Westerberg Explosion Test Vessel at the Underwriters Laboratories, Inc. in Northbrook, Illinois (UL). This apparatus was designed to compare new (unclassified) flammable gases and vapors with materials that had been classified into one of the four groups before development of this apparatus. The apparatus is used to determine the MESG and explosion pressures under various test conditions. The smallest MESG and largest explosion pressure under any of the several test conditions have been used as a basis for classification of the flammable gas or vapor. The four materials currently considered representative of Groups A, B, $\mathrm{C}$, and $\mathrm{D}$, their maximum explosion pressures, and minimum MESG's as determined in the Westerberg Explosion Test Vessel, are given in Table 1. The minimum ignition temperature and explosive range are also given. It should be noted that these values are comparative values only. Changes in the configuration of the test apparatus or the test conditions can change these values.

When a flammable gas or vapor not listed in Article 500 of the National Electrical Code is to be classified, the material can be tested in this apparatus and the minimum MESG and maximum explosion pressure of the unclassified material can be compared to the "base" materials in Groups A, B, C, and D given in Table 1. Once the new material is classified, selection of the proper type of explosion-proof electrical apparatus or enclosure is possible. Explosionproof apparatuses in the United States have been tested for one or more of these four groups of materials, using the "base" test material or a material representative of this test material.

$\Lambda$ similar method of classification of flammable gases and vapors is used inter- 


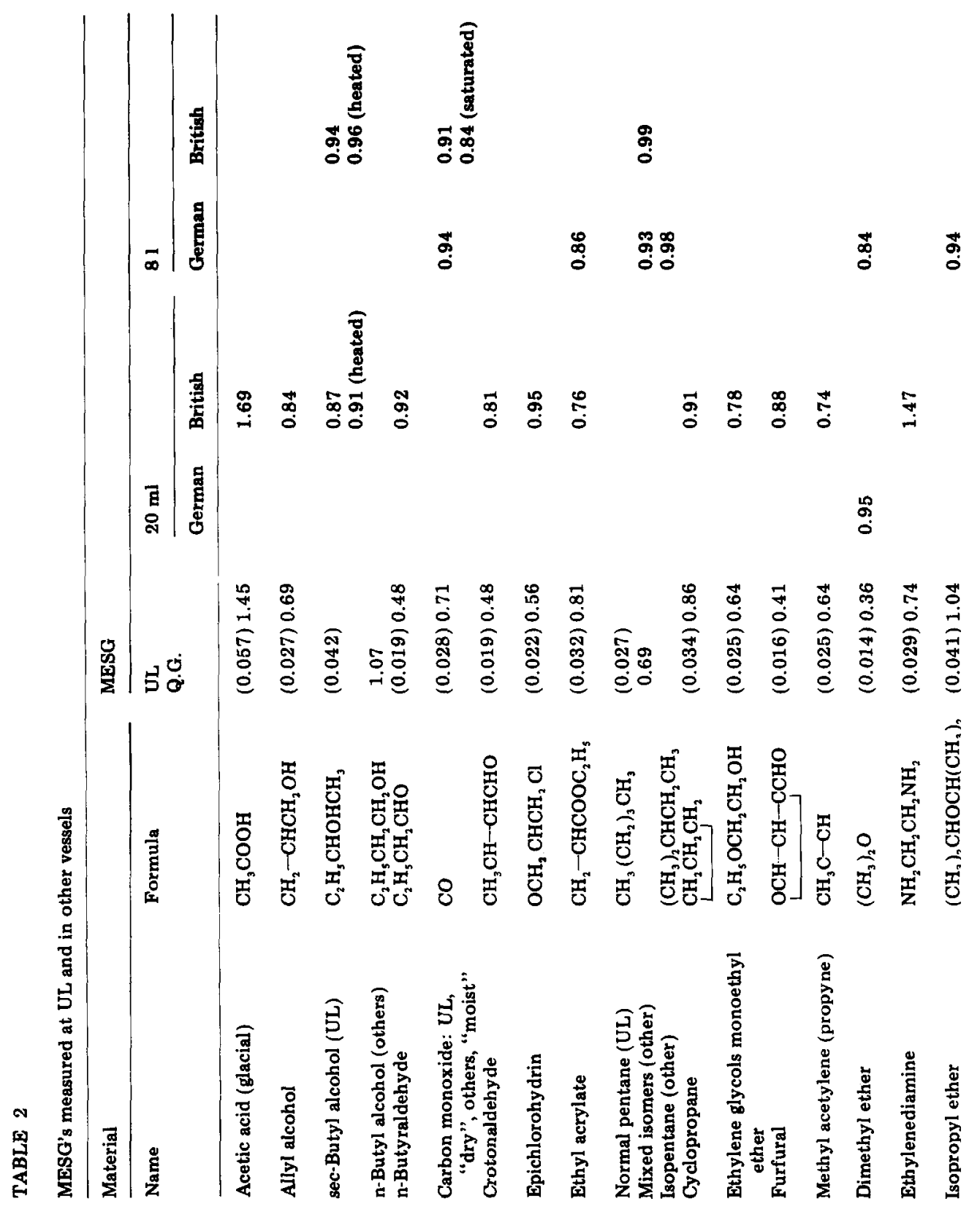




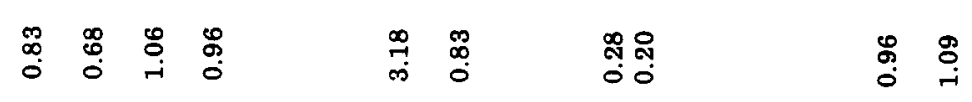

๘

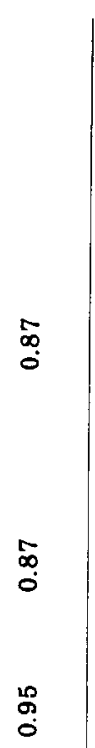

영 형

\%

ळ̆

옹

$\stackrel{8}{\circ} \quad 8$

용

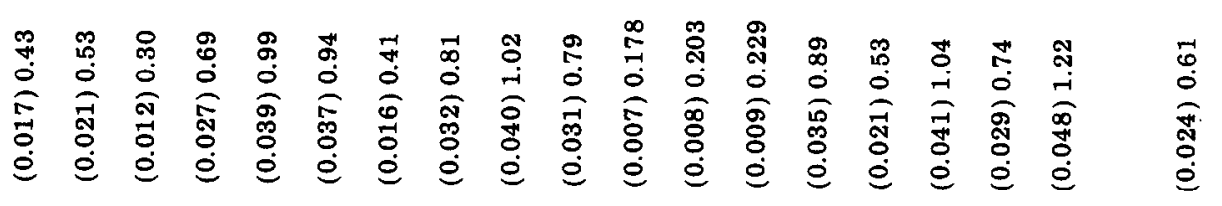

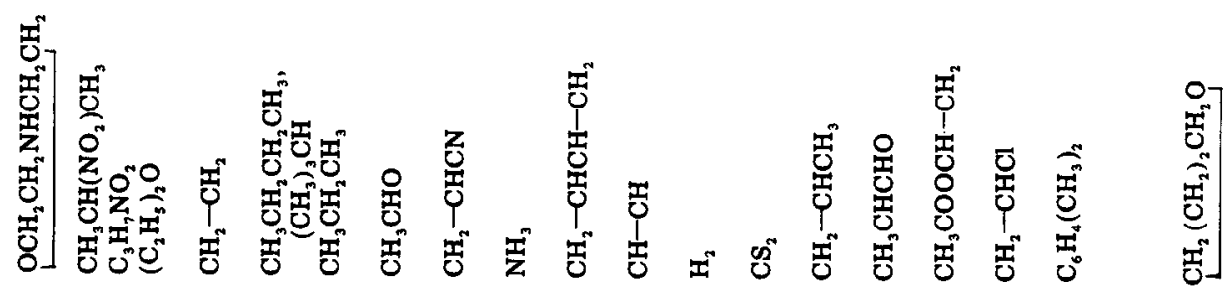

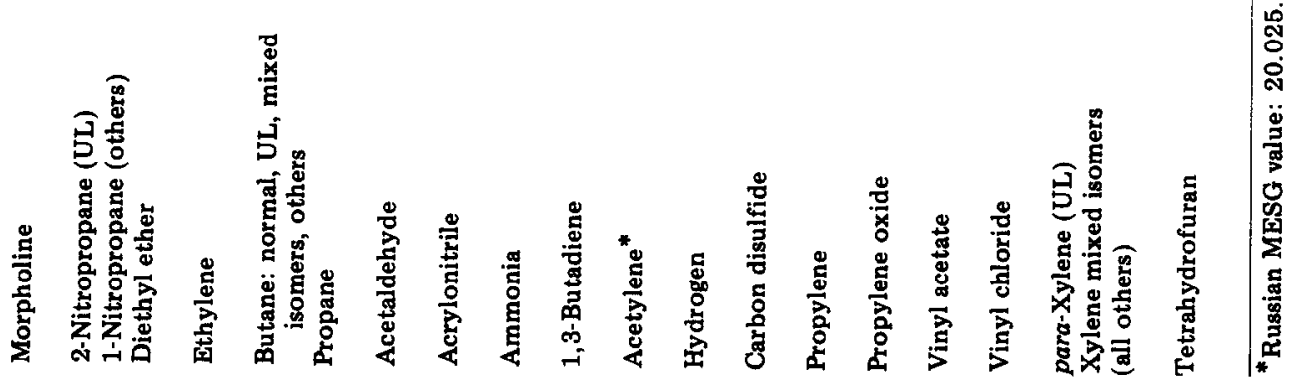


nationally, except that outside of North America classification is usually based solely on MESG (explosion pressures are not considered) and the measured MESG does not take into consideration changes in the MESG that may occur as a result of non-steady gas dynamic phenomena, usually called pressure piling, or turbulence of the mixture before ignition. As explained below the construction of the test apparatus also differs.

\section{Statement of the problem}

The anomaly being investigated was discovered a few years ago when it was noted that the United States' safe gap apparatus (the Westerberg apparatus at Underwriters Laboratories) yielded safe gap measurements that did not correlate well with those measured on other apparatuses, primarily in the United Kingdom and West Germany. The panel was charged with investigating this anomaly in some depth in May 1976.

During the intervening months, panel members J. Arthur Nicholls and Roger A. Strehlow visited Peter J. Schram at the Underwriters Laboratories, studied literature on the subject, and performed a simplified theoretical analysis. The latter serves to point up some of the probable causes of the observed differences from apparatus to apparatus. This report presents these observations and some preliminary conclusions and recommendations for further work. It is hoped that this study serves to define a research program that will ultimately lead to a sound engineering understanding of the testing technique, thereby allowing for improvement of the classification methods and, hence, greater confidence in the classification proposed for various substances.

\section{The anomaly}

Three basic apparatuses are used to determine the MESG of different specific compounds. These are the UL (Westerberg) apparatus in the United States [2], the 20-milliliter vessels [3] in the United Kingdom and West Germany, and the 8-liter vessels [3] in the United Kingdom and West Germany. The anomaly found is that, even though there are only slight differences between the MESGs measured in the 20-milliliter and 8-liter vessels, UL MESG values for the same compound using a quiescent atmosphere in the enclosure are sometimes only one-third as large as those measured in the 20-milliliter and 8-liter vessels [4]. Furthermore, since one of these anomalous compounds is diethyl ether, the United States standard compound used as a reference to separate the National Electrical Code Group B and Group C classifications, it is important to determine the cause of the differences.

Thirty-six compounds were found that have been tested by UL and in either or both of the 20-milliliter and 8-liter vessels [3,5]. These compounds are listed in Table 2 with their respective MESG values. A comparison of U.S. data and data generated in other vessels is shown in Fig.1. Table 3 lists the compounds in rough groupings by the ratio of the UL MESG to other MESGs. It is 


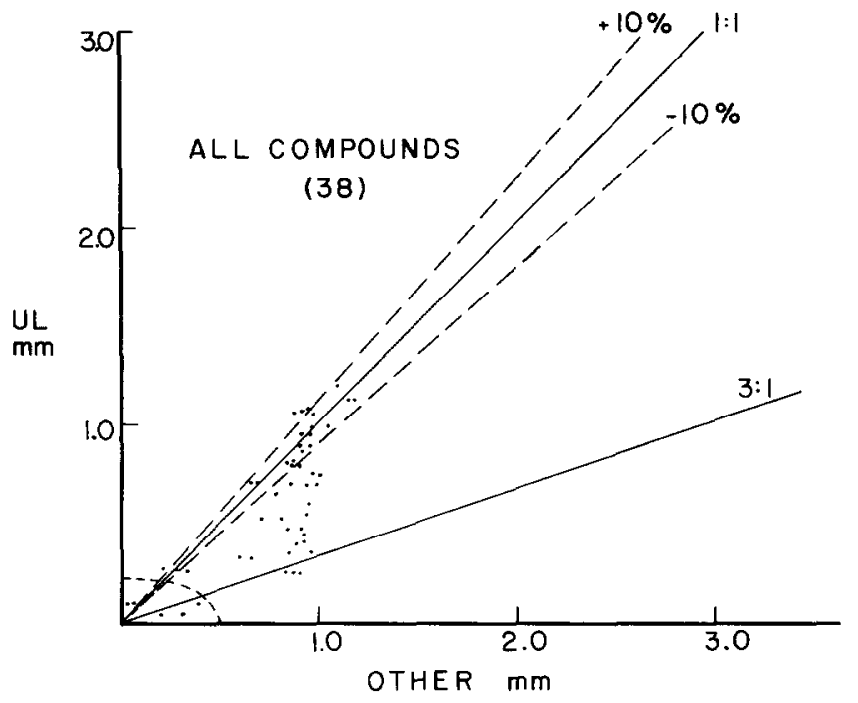

Fig.1. Comparison of UL and other measured MESG's for 36 compounds.

\section{TABLE 3}

Categories of compounds by anomalous behavior (ratios are (other MESG's/UL MESG's))

\section{Hydrocarbons}

$(C-H$ only $)(1: 1)$

$\begin{array}{ll}\text { Hydrogen } & \text { Acetylene* } \\ \text { Methane } & \text { Methyl acetylene } \\ \text { Propane } & \text { Propylene } \\ \text { Butane } & \text { Cyclopropane } \\ \text { Pentane } & \text { Butadiene } \\ \text { Ethylene } & \text { Xylene }\end{array}$

\section{1:1.5 Compounds}

Carbon monoxide

Propylene oxide

Ethylene oxide

Allyl alcohol

Ethylene glycol monoethyl ether

Tetrahydrofuran

Epichlorohydrin

Vinyl chloride

Nitropropane

\section{"Other" $1: 1$ compounds}

Isopropyl ether

sec- vs. iso-butyl alcohol

Vinyl acetate

Acetic acid

Ethyl acrylate

Acrylonitrile

\section{1:2 Compounds}

Acetaldehyde

Crotonaldehyde

n-Butylaldehyde

Ethylene dichloride

Ethylene diamine

Morpholine

Furfural
Maverick (1:3) compour

Ethyl ether

Methyl ether

Acetylene

Carbon disulphide

Ammonia

*Acetylene is the only hydrocarbon tested which is not a $1: 1$ compound. 
interesting to note that:

1. The UL-measured MESG is generally the same or smaller than those measured in the other apparatuses. (Some are slightly larger, but never more than 10\% larger; see Fig.1.) Since both the 20-milliliter and 8-liter vessels have a 25-millimeter surface length for the "gap" and the Westerberg apparatus has a surface length that is approximately $75 \%$ of this value, a very slightly smaller MESG could be anticipated for tests in the Westerberg apparatus, but not to the extent noted in the experiments.

2. All the hydrocarbons ( $\mathrm{CH}$ compounds) for which data are available have a $1: 1$ ratio within $\pm 10 \%$ (see Fig.2) except acetylene, which is $1: 2$.

3. The anomalous compounds ( $1: 3$ compounds) consist of the ethers (with the exception of isopropyl ether, which is a $1: 1$ compound) and the unusual compound, ammonia.

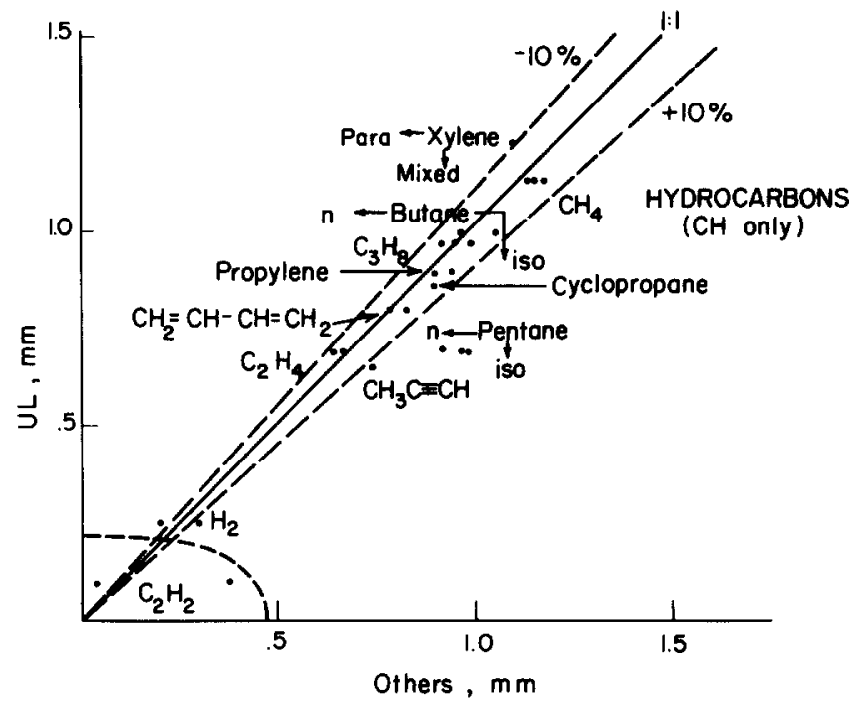

Fig. 2. UL and other measured MESG's for all hydrocarbons (HC compounds only).

Physics of the process and current theory

Observations have shown [6-9] that the following sequence of events occurs when a MESG experiment is performed with a quiescent gas in the enclosure. A spark ignites a flame in the enclosure and as the flame propagates the pressure rises, first slowly, and then more rapidly. Gas starts to escape through the gap. At first this is cold unburned gas, but later the flame reaches the gap and thereafter hot combustion products are exhausted into the surrounding atmosphere. When the gap is long, it may take a long time for combustion product gases to reach all parts of the gap. As soon as any product gases reach any portion of the gap, the mass flow-rate changes markedly.

If the pressure in the primary chamber where ignition occurred becomes sufficiently high (approximately twice that of the secondary vessel on the 
downstream side of the gap) the flow on the downstream side of the gap attains sonic velocity. If the pressure in the primary chamber markedly exceeds that required for sonic flow, the ouside flow becomes an underexpanded supersonic jet and shock waves are generated that ultimately cause the jet to become subsonic. In any event, during the entire time that combustion projects are entering the secondary chamber, the hot jets are mixing with the gases in that chamber. It has been observed that at gap separations slightly above the MESG value, and after some delay time, ignition of the gas in the secondary chamber occurs some distance from the gap, in the mixing region along the jet boundaries, where the hot combustion products are mixing with the surrounding cold flammable mixture.

Phillips [6-9] has written extensively on the subject and has proposed a theory for the ignition process in which he assumes that a quasi-steady jet is issuing from the gap. His theory is essentially a "thermal explosion" theory in which a temperature-time curve is developed for the mixing region in the secondary chamber. In simple words, if the gap is less than the MESG, then losses due to heat transfer to the wall of the gap and mixing with unburned flammable gases in the secondary chamber dissipate the heat generated by the exothermic reactions in the mixing regions of the secondary chamber. As the gap is assumed to be larger, there is at first a slight elevation of the calculated temperature-time curve. However, at some critical gap size (the MESG) the calculated temperature-time curve first drops and then shows a dramatic rise due to an "explosion" of the flammable portion of the mixture in the secondary chamber. The theory is quite analogous to the thermal theory for homogeneous vessel explosions, at least in its general structure.

The theory contains a number of more or less adjustable constants, such as the pre-exponential and Arrhenius constants for the overall exothermic chemical reactions, heat transfer coefficients, and mixing coefficients. Within this framework, Phillips has claimed to calculate correct MESGs for such diverse fuels as carbon disulfide, ammonia, methane, hydrogen, and ethylene.

Some serious oversimplifications have been made by Phillips in deriving his theory. This statement is based on the following observations:

1. Some compounds exhibit very long delay times to ignition as MESG is approached. Canadian workers [10] have determined that diethyl ether has a delay time of about $\mathbf{4 0 0} \mathrm{ms}$ in an apparatus with dimensions similar to those of the UL apparatus. Phillips comments that methane exhibits a delay to ignition of only $5 \mathrm{~ms}$. In view of this range of delay time, the quasi-steady-state assumption may not be universally valid.

2. The hot gas will contain relatively large quantities of reactive materials, since it is a post-flame gas. Thus, it is difficult to see how a single thermal theory could yield a correct MESG for the wide range of fuels mentioned above. Of the compounds listed, those that are similar kinetically are the higher hydrocarbons with no other attached atoms. Thus, it appears that the theory would be improved by taking into account (a) the effect of transient flow behavior and (b) the effect of reactive radicals from the hot gas. 
3. The Phillips treatment of the mixing process in the secondary chamber is overly simplified.

4. The theory has not taken into consideration some very important aspects. For example, it has neglected the size of the primary and secondary chambers and the total open area of the gap. These geometric features strongly affect the characteristic times for flame travel, pressure buildup, pressure decay, and ignition delay.

\section{Apparatus differences}

It is obvious that the anomaly exists because the MESG is an apparatus-dependent quantity. Thus, differences among the various forms of the apparatus must be investigated. Table 4 lists the important characteristics of the three standard MESG apparatuses. Fig. 3 shows the different gaps to scale. It can be seen that the most apparent differences from vessel to vessel are the characteristic combustion times $\left(t_{\mathrm{c}}\right)$ and the $L^{*}$ of the vessels.

The characteristic combustion times were estimated for a closed spherical chamber with central ignition using the technique of Bradley and Mitcheson [11] and an assumed normal burning velocity of $0.40 \mathrm{~m} / \mathrm{s}$. Note that they differ by an order of magnitude.

$L^{*}$ is a measure of the characteristic exhausting time of a pressurized vessel

\section{TABLE 4}

Characteristics of MESG apparatuses

\begin{tabular}{|c|c|c|c|}
\hline \multirow[t]{2}{*}{ Characteristic } & \multicolumn{3}{|l|}{ Vessel } \\
\hline & UL & 81 & $20 \mathrm{ml}$ \\
\hline $\begin{array}{l}\text { Enclosure volume } \\
\text { Shape }\end{array}$ & $\begin{array}{l}28,315 \mathrm{cc} \\
\text { Cylindrical }\end{array}$ & $\begin{array}{l}8000 \text { ce } \\
\text { Spherical }\end{array}$ & $\begin{array}{l}20 \mathrm{cc} \\
\text { Spherical }\end{array}$ \\
\hline $\begin{array}{l}\text { Combustion time }\left(t_{\mathrm{c}}\right) \\
\text { no vent, central ignition } \\
L^{*}=V / A(\mathrm{~cm}) \text { for a gap }\end{array}$ & $137.1 \mathrm{~ms}$ & $89.97 \mathrm{~ms}$ & $12.18 \mathrm{~ms}$ \\
\hline of $1 \mathrm{~mm}$ & 26713 & 1282.5 & 19.05 \\
\hline$L * t_{\mathrm{c}}(\mathrm{cm} / \mathrm{ms})$ & 195 & 14.3 & 1.56 \\
\hline $\begin{array}{l}\text { Gap length in direction } \\
\text { of flow }\end{array}$ & $\begin{array}{l}31 \mathrm{~mm} \text { top } \\
18.7 \mathrm{~mm} \text { bottom }\end{array}$ & $25 \mathrm{~mm}$ & $25 \mathrm{~mm}$ \\
\hline Breadth of gap & $\begin{array}{l}\text { Flat surface } \\
4^{\prime \prime} \text { long }\end{array}$ & Circumferential & Circumferential \\
\hline Location of ignition spark & Near gap & $\begin{array}{l}\text { Well off center } \\
\text { near one portion } \\
\text { of gap }\end{array}$ & Central \\
\hline $\begin{array}{l}\text { Size of receptor vessel } \\
\text { relative to enclosure } \\
\text { vessel }\end{array}$ & $1: 1$ & $>1: 1$ & $>10: 1$ \\
\hline
\end{tabular}

Note: $V=$ volume of primary vessel, $A=$ gap area. 


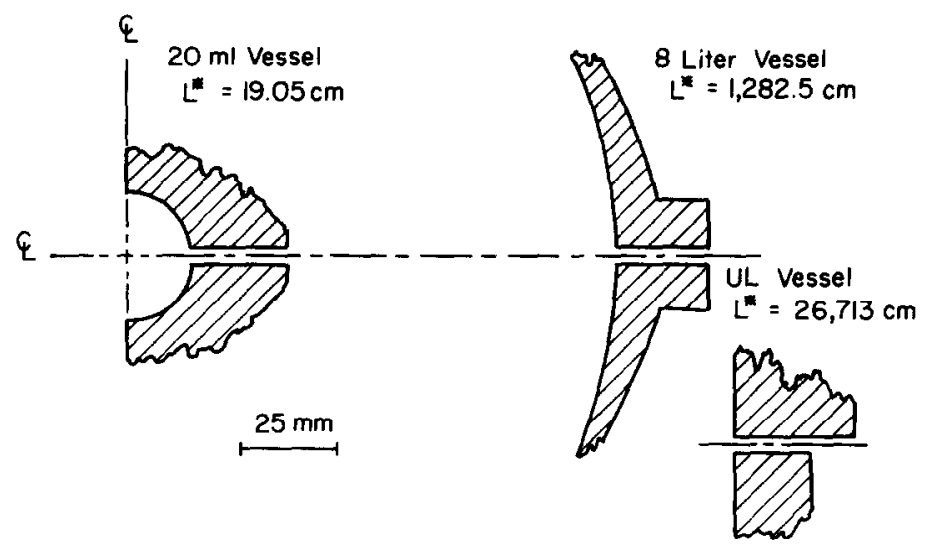

Fig. 3. Scale drawing of the gaps and volumes in the three MESG apparatuses.

with a hole in its side. It is equal to the volume, $V$, of the vessel divided by the area, $A$, of the hole. If two vessels with different $L^{*}$ values are pressurized with the same gas they will attain any specified lower pressure (still above atmospheric) at a time after the hole is opened according to the relation:

$t_{1} / t_{2}=L^{*}{ }_{1} / L^{*}{ }_{2}$

Thus $L^{*}$ is directly proportional to the exhausting time, if all else is held constant (initial conditions, heat loss, etc.). The $L^{*}$ values of these vessels differ by over three orders of magnitude. It will be noted that the ratios of $L^{*}$ vary

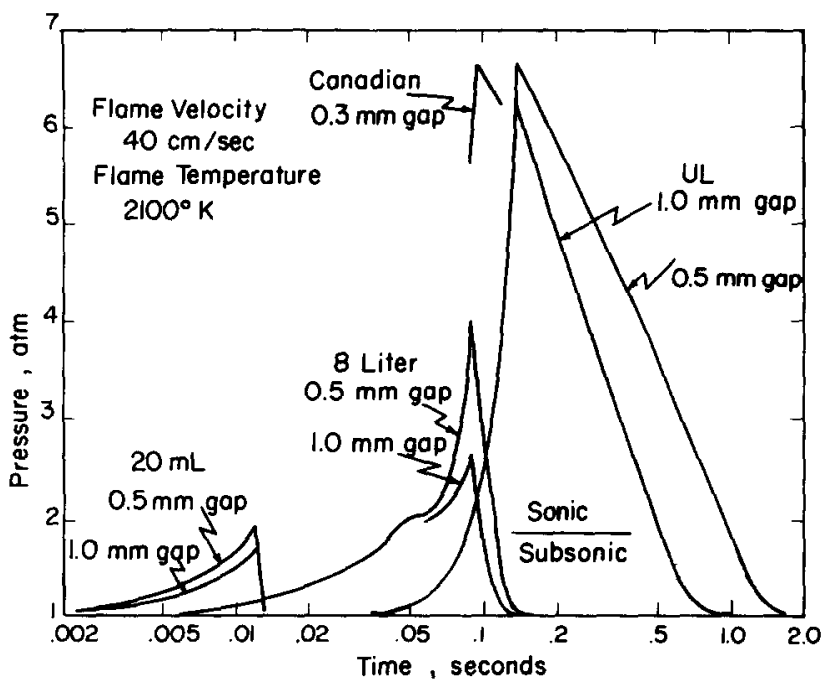

Fig.4. Pressure-time histories in the three vessels for two different gap widths. A calculation for the Canadian [10] vessel is also included. 
as the combustion time differences. That is, the UL vessel not only has a slower rate of pressure rise, it also has a slower rate of pressure decay.

Another important ratio is $L^{*} / t_{\mathrm{c}}(\mathrm{cm} / \mathrm{ms})$. This quantity determines the maximum pressure rise in the enclosure during an experiment. To evaluate the effect semi-quantitatively, a very simple approximate theory for the transient venting of a vessel containing a flame and a sharp-edged orfice in its wall was coded on a computer to determine the pressure-time relationship for these different vessels. The results of this simplified calculation are shown in Fig.4. Note that the UL vessel, which has an $L^{*} / t_{\mathrm{c}}$ of 19.05 , reaches a calculated transient pressure at the end of combustion of almost 7 atmospheres (assuming no venting or heat losses). When $L^{*} / t_{\mathrm{c}}$ is reduced to 14.3 , the calculated maximum pressure drops to about 3 atmospheres, and when $L^{*} / t_{\mathrm{c}}$ drops to 1.56 , the calculated maximum pressure is only about 1.7 atmospheres. At this pressure, the flow through the gap would never become sonic. Thus we see that the variation in $L^{*}, t_{\mathrm{c}}$, and $L^{*} / t_{\mathrm{c}}$ for the three "standard" vessels causes marked differences in (a) the total time available for ignition of the external flammable mixture ( 2 orders of magnitude variation), (b) the pressure levels generated in the enclosure (a factor of about 7 in $\Delta P$ ), and (c) the velocity of the flow through the gap (the 20-milliliter vessel never reaches sonic flow).

There are three other major differences from apparatus to apparatus that are not as dramatically obvious but still may individually or collectively contribute to the anomaly. The first difference relates to the position of the spark relative to the location of the gap. Spark position is critical for two reasons. In the first place, the mass flow through the gap is strongly dependent on the stagnation temperature of the gas; when the flame reaches the gap, the mass flow through the gap will drop, thus changing the total exhausting time. Also, if the flame reaches the gap early there will be more time for the hot gases to mix with and possibly ignite the receptor gases. In the three vessels of interest, the spark-gap relationships are quite different. In the UL apparatus the gap is short and for those tests used in this comparison the spark is near it. Thus, during most of the exhausting time the gap will be exhausting hot combustion products. In the 8-liter vessel the spark is mounted near one part of the gap, which is circumferential. Thus, while part of the gap exhausts hot gases early, the remainder of the gap exhausts cold gas. In contrast, ignition in the 20milliliter vessel is central, and a large fraction of the initial contents must be exhausted as unburned gas before the flame reaches the gap.

The second major difference pertains to the geometry of the gap itself. The UL apparatus is unique because the lengths of the lower and upper surfaces of the gap are quite different. This asymmetry of the gap will have an effect on the mixing process in some unknown way, particularly when the pressure ratio across the gap is larger than the actual pressure ratio for sonic flow. On the other hand, the gap in the 20-milliliter vessel is unique because of its small radius of curvature. The external circumference of this gap is 2.5 times longer than the internal circumference. This means that if this gap were ever to reach a critical flow pressure ratio, the location of the sonic surface would probably differ from that in the 8-liter or UL vessels. 
The third major difference concerns the relative size of the test vessel and receptor vessel. In practice the receptor vessel is the flammable atmosphere in the room itself, and therefore for proper testing the receptor vessel should generally be much larger than the test vessel. This is true for the 20-milliliter vessel test apparatus. We have no data for the 8-liter test apparatus, but it appears that the receptor vessel is slightly larger than the 8-liter vessel. In the Westerberg apparatus the test vessel is slightly larger than the receptor vessel. This means that during the performance of a test the gases that are initially in the receptor vessel will be heated to a considerable extent by adiabatic compression processes. This could cause spurious ignition with a smaller gap because the hot compressed gas would be easier to ignite.

\section{Differences from compound to compound}

The major differences among compounds are due to differences in the kinetics of the exothermic oxidation processes and differences in flame temperatures. There is some evidence for this in the statement by Phillips [6-9] that methane (a "regular" compound) takes only $5 \mathrm{~ms}$ to ignite at gap width slightly larger than the MESG while the Canadian work [10] showed a 400-ms delay for diethyl ether under the same circumstances. The Canadian measurements also showed that the delay to ignition becomes shorter as the gap becomes larger. This is shown in Fig.5, a schematic representation of delay versus gap width. Two curves of ignition delay versus gap width are shown in Fig. 5 . These curves are idealized in that it is assumed that the time at which the flame reaches the gap is known and that the delay is zero if the gap width is equal to the quenching distance, $d_{0}$. The lower curve, labeled "maverick" compound, is a schematic representation of the behavior found by the Canadian investigators [10].

Fig. 5 is also intended to illustrate a possible result of kinetic rate and flame temperature differences among compounds. It is highly likely that the different

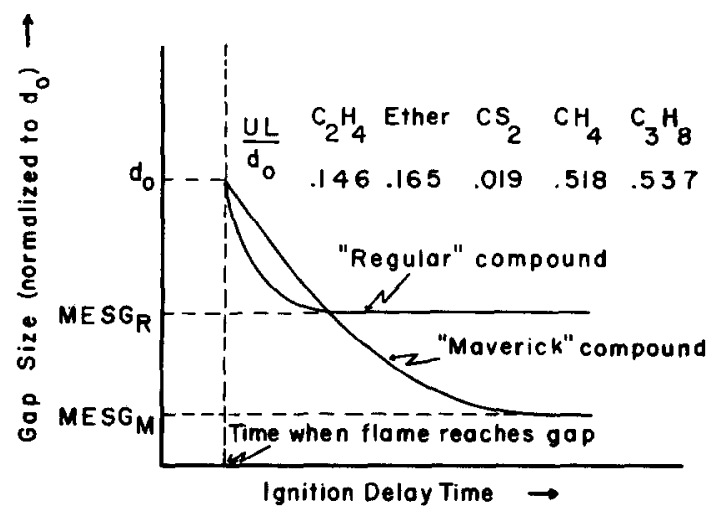

Fig.5. Schematic of the time delay to ignition-gap width relationship for two compounds with markedly different kinetics. 
types of ignition delay-gap width behaviors are related to the compound tested. One would expect a curve like that labeled "regular" compound to exhibit only small MESG differences from vessel to vessel because ignition occurs rapidly, even near the MESG, and the different vessel times illustrated in Fig.4 become unimportant. However, if the ignition delay time-gap curve corresponded to the "maverick" compound curve in Fig.5, sizable differences in MESGs could be expected among test vessels. These differences in behavior must be controlled primarily by kinetic rate and flame temperature differences.

Another observation of differences is summarized in Fig.5. The ratio of $\mathrm{UL} / d_{0}$ tabulated on this figure is the ratio of the UL MESG to the parallel plate quenching distance. The parallel plate quenching distance is the maximum plate separation that will just prevent a flame from propagating through a quiescent mixture. It would, of course, be expected that measured MESG's would be smaller than $d_{0}$ because in the MESG apparatus gases are blown through the gap. Note the large differences in this ratio for the few compounds tabulated on Fig. 5. This ratio appears to be sensitive to kinetics and flame temperature of individual compounds.

\section{Recommendations}

It has been shown that the large differences among the MESG apparatus used throughout the world can, in some cases, have significant effects on the results obtained. Therefore, the hazard classification of chemical compounds depends on the experiment performed. Considering that OSHA is currently interested in classifying 400 compounds, and undoubtedly there will be more in the future, a good engineering assessment of the classification process is needed. It is impractical to test each one of the substances. Yet, even if this were done, the unknown influence of the apparatus would preclude complete confidence in the results obtained. For this reason we recommend that a combination theoretical-experimental research study of the MESG problem be conducted.

The type of study envisioned is not one that exhaustively treats the details of a given compound and apparatus, but rather one that represents the physical and chemical properties of some general compound and the geometrical aspects of the test apparatus as parameters of the problem. In this parametric sense, the analysis would consider the size and shape of the primary chamber and venting slot, the size and shape of the secondary chamber, the flame speed (with generalized correction for pressure and temperature effects), transport characteristics for the mixing process, and concentration and activation energy representation, albeit with justified simplification. Values for the various parameters would be prescribed when desired. Further, it is expected that some experiments would have to be conducted in order to remove certain assumptions in the theory and also to validate the final predictions by data collected on a few appreciably different compounds.

The objective of this study would be to answer the following questions: 
(1) Can MESG determinations in the standard International Electrotechnical Commission (IEC) test apparatus be used either directly, or with some adjustment, for classifying materials in the United States, taking into account differences in installation practices?

(2) Can the IEC test apparatus be modified to lead to test methods and data that are valid for both cable and conduit systems?

(3) Can the important ignition parameters of materials be identified in a way that allows increased confidence in classification without testing?

\section{References}

1 National Flectrical Code, Article 500, NFPA No. 70 (ANSI-C1), 1975.

2 Underwriters Laboratories, Inc., An investigation of fifteen flammable gases or vapors with respect to explosion-proof electrical equipment, Bulletin of Research 58, Northbrook, IL, August 1969.

3 G. A. Lund and H. Phillips, A summary of experimental data on the maximum experimental safe gap, SMRE Report R2 (T 7403277), Department of Trade and Industry, Safety in Mines Research Establishment, Red Hill, Sheffield, United Kingdom, 1973.

$4 \mathrm{H}$. Carhart and D.L. Katz, Fire hazard classification of chemical vapors relative to explosion-proof electrical equipment, Final Panel Report, Contract TGC-15559-A, Project 713192 , U.S. Coast Guard, Office of Research and Development, February 10, 1970, Figure 7.

5 Underwriters Laboratories, Inc., An investigation of additional flammable gases with respect to explosion-proof electrical equipment, Bulletin of Research $58 \mathrm{~A}$, Northbrook, IL, June 1976.

$6 \mathrm{H}$. Phillips, Theory of suppression of explosions of narrow gaps, Industrial and Chemical Engineering Symposium, Series 33, 1972, pp. 17-24.

$7 \mathrm{H}$. Phillips, A non-dimensional parameter chemistry mixing process in a model of thermal gas ignition, Comb. and Flame, 19 (1972) 181.

$8 \mathrm{H}$. Phillips, Ignition in a transient turbulent jet on hot inert gas, Combust. Flame, 19 (1972) 187.

$9 \mathrm{H}$. Phillips, On the Transmission of an Explosion Trough a Gap Smaller Than Quenching Distance, Combust. Flame, 7 (1963) 129.

10 G.K. Brown, E.D. Dairty and A. D'Aoust, The Variation of Maximum Experimental Safe Gap with Secondary Explosion Chamber Relief for Ether-Air Mixtures, Division Report FRC 71/53, CEAL 242, Department of Energy, Mines and Resources, Ottawa, Canada, August 1971.

11 D. Bradley and A. Mitcheson, Mathematical solutions for explosions in spherical vessels, Combust. Flame, 26 (1976) 201. 